\title{
Original
}

\section{The Use of Neural Networks as Numeric-to-Symbolic Converters for Adaptive Control Systems}

\author{
by \\ ${\text { Goro } \text { ObINata }^{\dagger} \text {, Yasushi Muragishi }}^{\dagger \dagger}$, and Yuichi TaKeI $^{\dagger}$
}

\begin{abstract}
A new control scheme is proposed in this paper which can cope with varying environment as results of load disturbances, changes of plant dynamics, and failures of components. The objective of this paper is to blend numeric-tosymbolic conversion techniques with linear conventional controllers so as to adapt to the varying environment of the system. The control scheme is based on the parametrization of stabilizing controllers, which is called Kucera/Yula parametrization. The parametrization has been extended to the class of systems which contain numeric-to-symbolic converters. It is shown how the numeric-to-symbolic converters can be blended with the linear controllers.
\end{abstract}

Key Words : Adaptive Control, Neural Network, Numeric-to-Symbolic

Converter, Parametrization, Pattern Classification, Control

System Design

\section{Introduction}

A new control scheme is proposed in this paper which can cope with varying environment as results of load disturbances, changes of plant dynamics, and failures of components. Many adaptive control methods have been applied to the systems with varying environment. There are several problems in use of adaptive control techniques ; for examples, burst behavior from wrong assumptions $^{1)}$, lack of proof in convergence of algorithms ${ }^{2)}$, chaotic phenomena from nonlinearity ${ }^{3)}$. Another relatively new idea for adaptation to varying environment is to give much attention on event-driven problems in control systems $s^{4}$. In most of actual plants, the change of plant parameters occurs only at discrete points in time in response to the failure of the components. The disturbance comes into the system at the time when the particular event occurs. If we know how the failures have effects on the plant dynamics; we can tune the controller parameters so as to keep the performance, or to suppress the deterioration. Therefore, incorporation of human intelligence into control systems has been considered here, which is classified into heuristic control schemes. Fuzzy theory and neural networks can be used for such a purpose, and implemented easily in digital computers $^{5), 6)}$. While many applications of these heuristic approaches have been realized, linear

$\dagger$ Department of Mechanical Engineering, Mining College, Akita University Akita 010 Japan

$\dagger \dagger$ Research \& Developement Laboratory Shinko Electric Co. Ltd Ise 516 Japan 
controllers still keep important roles in industrial processes. In most of actual situations, changes of environment occur in the systems which are controlled by the linear controllers. So, it is desirable to blend heuristic decision techniques with linear controllers.

The objective is to blend numeric-to-symbolic conversion techniques with linear controllers so as to adapt to the varying environment of the systems. The numeric-to-symbolic converter is to cassify the change of the plant dynamics based on the heuristic rules. Tuning of the controller is carried out under instructions from the output of the converter. The tuning method is based on the parametrization of the class of all stabilizing controllers ${ }^{7)}$. The parametrization is in terms of an arbitrary proper stable transfer function, and has been extended to the class of systems which contain numeric-to-symbolic converters. A neural network with back propagation training rule is used as the numeric-to-symbolic converter. The numeric-to-symbolic converter is trained in closed-loop with prior knowledge about varying environment. The proposed control scheme is different from usual supervisory control scheme because there is no hierarchical structure. It is shown how the numeric-to-symbolic converters can be blended with the linear controllers and the proposed control scheme improves the adaptability of the hybrid control systems. Simulation examples are given to illustrate the efficiency of the proposed control scheme.

\section{Class of all stabilizing controllers}

In this section, background theory is organized. Given a linear plant with nominal state space description

$$
\dot{\mathrm{X}}=\mathrm{Ax}+\mathrm{Bu}, \quad \mathrm{y}=\mathrm{Cx}
$$

and transfer function

$$
\mathbf{P}(\mathbf{s})=\mathbf{C}(\mathbf{s I}-\mathbf{A})^{-1} \mathbf{B} \longleftrightarrow\left[\begin{array}{l|l}
\mathbf{A} & \mathbf{B} \\
\hline \mathbf{C} & \mathbf{O}
\end{array}\right]
$$

We assume that $\mathrm{K}(\mathrm{s})$ is a stabilizing controller characterized in terms of $\mathrm{F}, \mathrm{H}$ as

$$
\mathbf{K}(\mathbf{s}) \longleftrightarrow\left[\begin{array}{c|c}
\overline{\mathrm{A}} & \mathbf{H} \\
\hline-\mathbf{F} & \mathbf{O}
\end{array}\right]
$$

where

$$
\overline{\mathrm{A}}=\mathrm{A}+\mathrm{BF}+\mathrm{HC}
$$

It is clear that $\mathrm{K}(\mathrm{s})$ can be designed as an LQG controller. Let us define

and

$$
\left[\begin{array}{ll}
\mathbf{D}(\mathbf{s}) & \mathbf{U}(\mathbf{s}) \\
\mathrm{N}(\mathbf{s}) & \mathrm{V}(\mathbf{s})
\end{array}\right]=\left[\begin{array}{ll}
\mathbf{I} & \mathbf{O} \\
\mathbf{O} & \mathbf{I}
\end{array}\right]+\left[\begin{array}{l}
\mathbf{F} \\
\mathbf{C}
\end{array}\right](\mathbf{s I}-\mathbf{A}+\mathbf{B F})^{-1}\left[\begin{array}{ll}
\mathbf{B} & \mathbf{H}
\end{array}\right]
$$

$$
\left[\begin{array}{rr}
\widetilde{V}(\mathbf{s}) & -\widetilde{U}(\mathbf{s}) \\
-\widetilde{D}(\mathbf{s}) & \widetilde{N}(\mathbf{s})
\end{array}\right]=\left[\begin{array}{ll}
\mathbf{I} & \mathbf{O} \\
\mathbf{O} & \mathbf{I}
\end{array}\right]+\left[\begin{array}{c}
-\mathbf{F} \\
\mathbf{C}
\end{array}\right](\mathbf{s I}-\mathbf{A}-\mathbf{H C})^{-1}\left[\begin{array}{ll}
\mathbf{B} & \mathbf{H}
\end{array}\right]
$$

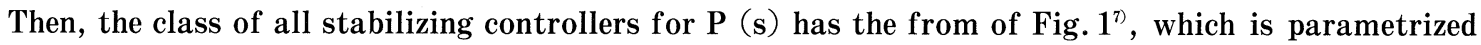
in $\mathbf{Q}(\mathbf{s}) \in \mathrm{RH}^{\infty}$. The $\mathbf{J}(\mathbf{s})$ is defined as follows :

$$
\mathbf{J}(\mathbf{s})=\left[\begin{array}{cc}
\mathrm{U}(\mathbf{s}) \mathbf{V}^{-1}(\mathbf{s}) & \widetilde{\mathrm{V}}^{-1}(\mathbf{s}) \\
& \\
\mathrm{V}^{-1}(\mathbf{s}) & -\mathrm{V}^{-1}(\mathbf{s}) \mathrm{N}(\mathrm{s})
\end{array}\right] \leftrightarrow\left[\begin{array}{c|cc}
\overline{\mathrm{A}} & \mathrm{H} & -\mathrm{B} \\
\hline-\mathrm{F} & \mathrm{O} & \mathrm{I} \\
\mathrm{C} & \mathrm{I} & \mathrm{O}
\end{array}\right]
$$


If $\mathrm{Q}(\mathrm{s})=\mathbf{O}$, then the controller for $\mathrm{P}(\mathrm{s})$ is $\mathrm{J}_{11}(\mathrm{~s})=\mathrm{K}(\mathrm{s})$. When there are some time-varying disturbances or time-varying perturbations of the plant dynamics, we can utilize free parameters $Q$ (s) of the augmented controller to reduce the effects of the disturbances on the performance. Tay and Moor ${ }^{8)}$ proposed a useful method in which $\mathrm{Q}$ (s) is adaptively tuned to reject the time-varying disturbances. However, in the case of perturbations of plant dynamics, we cannot ensure any longer the stability of the control system. Obinata and Moore $^{9)}$ gives a parametrization for the class of all stabilizing controllers for two or more plant dynamics. The result for stability has been extended to the case of time-varying $P$ and $Q^{10)}$. Optimal tuning of free parameters is difficult in the case. In this paper, we take an-

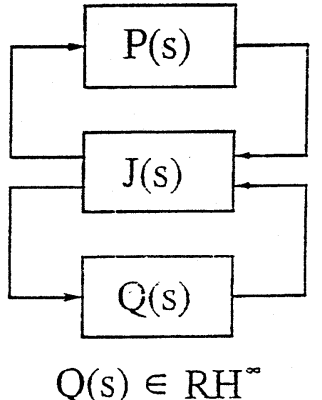

Fig. 1 Class of all stabilizing controller other approach for tuning $\mathbf{Q}(\mathbf{s})$, which is explored in subsequent sections.

\section{Classification of events using neural network in closed-loop}

We can find out a lot of event-driven problems in actual control systems ${ }^{4,11)}$. In most of actual plants, the change of plant parameters occurs in response to failure of the components. The disturbance comes into the system at the time when the particular event occurs. We may know the pattern of the changes or the characteristics of the disturbances corresponding to the event. In adaptive control schemes, the change of the dynamics has to be identified with accuracy. If we have prior knowledge about the pattern of the change, it is a relatively easy task to classify the event which causes the corresponding change. This is the point of our method which differs from conventional adaptive control scheme.

In this scction, a neural network is used to classify numeric data and assign symbols to various classes. The neural network considered here contains a single hidden layer between the input and the output layers, as illustrated in Fig. 2 for the case of four nodes at input and hidden level, and two nodes at output level. Assume that we know the several dynamics of the plant $\mathbf{P}_{\mathrm{oo}}, \mathbf{P}_{\mathrm{ol}}, \cdots \cdots$ which are determined and driven by the discrete events, and the controller $K_{o}$ for nominal plant $P_{o o}$ is

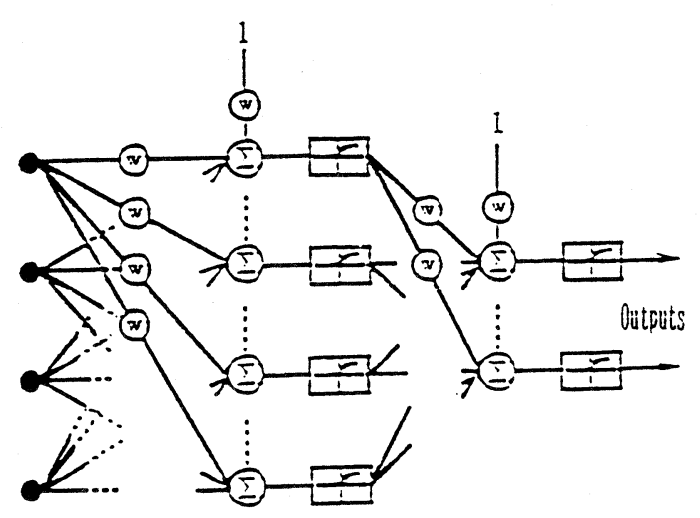

Input layer Bidden layer Gutput lajer

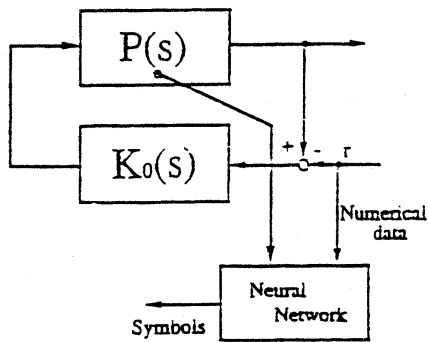

Fig. 3 Classification of events usigdata set $\mathrm{S} 1$ in closedloop

Fig. 2 Three-layer neural network 


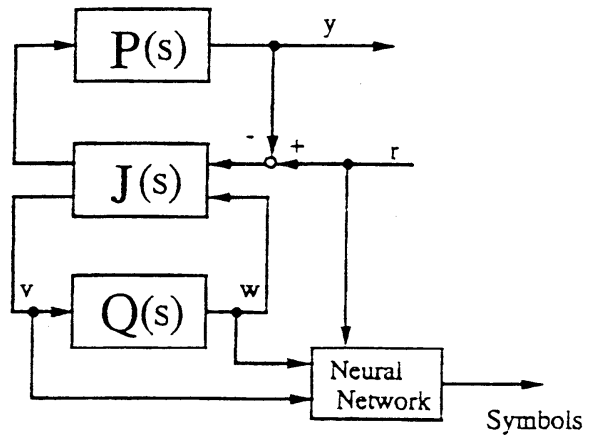

Fig. 4 Classification of events using data set $\mathrm{S} 2$ in closed-loop

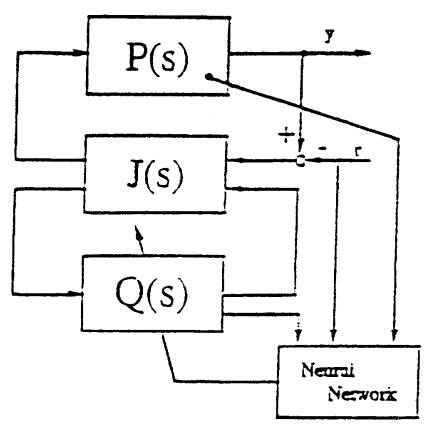

Fig. 5 Adaptive scheme using neural network

designed. Time histories of plant inputs, outputs and another signals corresponding to each dynamics can be obtained in the closed-loop system with the nominal controller. The following two sets of signals selection are considered here. S1: State variables of the plant, reference input to the control system and the derivatives of those signals are taken up for inputs of network, shown in Fig. 3. S 2 : Two variables $\mathrm{v}$ and $\mathrm{w}$ in the controller include an essential information of the changes since the transfer function from $\mathrm{w}$ to $\mathrm{v}$ is equivalent to that of the difference between the nominal plant $P_{o o}$ and the changed plant $P_{i j}$. Therefore, these variables $v$ and $w$, reference input $r$, and the derivative $\mathrm{r}$ are suitable for inputs of the converter, shown in Fig. 4.

For two cases: S 1 and S 2, the neural network is trained by back-propagation to classify the plant dynamics with the prior knowledge of the dynamics. The desired responses for the neural network are binary valued symbols which represent each dynamics. Therefore, the neural network in our method is used as a numeric-to-symbolic converter. After the training stage, we set the neural network in the closed-loop for detecting the change of plant dynamics. If the plant keeps the nominal dynamics, the output of the neural network indicates the corresponding binary valued symbol. When the change of the plant dynamics occurs, the corresponding symbol is obtained as an output of the neural network.

\section{Adaptation to the change of plant dynamics}

We can tune or change the controller based on the symbols given by the neural network. However, a sudden change of the controller is undesirable ; morever, it is difficult to guarantee the performance during the tuning stage. We will propose the method that the tuning part of the controller is combined to the fixed main controller as an additional loop. The structure comes from the parametrization of all stabilizing controllers, which is already mentioned in section 2 . So, the controller which represents the class of all stabilizing controllers, Fig. 1, is reorganized with the neural network as in Fig. 5. The output of the neural networks as a switch which selects suitable tuning part for the changed dynamics. Define the difference between the nominal plant and the changed plant by $\triangle \mathbf{P}_{\mathrm{i} j}(\mathrm{~s})=\mathbf{P}_{\mathrm{ij}}(\mathrm{s})-\mathbf{P}_{\mathrm{oo}}(\mathrm{s})$ and

$$
\mathbf{Q}_{\mathrm{p}}(\mathbf{s})=(\mathbf{I}-\mathbf{D}(\mathbf{s}) \triangle \mathbf{P}(\mathbf{s}) \mathbf{U}(\mathbf{s}))^{-1} \mathbf{D}(\mathbf{s}) \triangle \mathbf{P}(\mathbf{s}) \mathbf{D}(\mathbf{s})
$$


Then, we can check stability of the control system by the following lemma ${ }^{10)}$ : The control system is stable if and only if the closed-loop system composed by the selected tuning part $Q$ (s) and transfer function $Q_{p}$ (s) is stable. The sufficient condition of stability is well known as Small Gain Theorem ; so, it is easy to obtain the sufficient condition for stability on the tuning part $Q$ (s), which gives a merit in the use of the parametrization.

\section{Simulation results}

In this section, an example of how numeric-to-symbolic conversion can be done via the neural network is given. Consider the following simple SISO system with the transfer function :

$$
\mathbf{P}_{\mathrm{oo}}(\mathrm{s})=\mathbf{k}_{\mathrm{o}} /\left\{\mathrm{s}\left(1+\mathrm{T}_{\mathrm{o}} \mathrm{s}\right)\right\}, \quad \mathbf{k}_{\mathrm{o}}=1, \quad \mathbf{T}_{\mathrm{o}}=0.1
$$

The changes of the plant dynamics are taken into considerations as follows :

$$
\mathrm{P}_{\mathrm{ij}}(\mathrm{s})=\mathrm{k}_{\mathrm{i}} /\left\{\mathrm{s}\left(1+\mathrm{T}_{\mathrm{j}} \mathrm{s}\right)\right\}, \quad \mathrm{i}=\mathbf{0 . 1} \mathrm{j}=\mathbf{0 . 1}
$$

where the necessary information on $k_{i}, T_{j}$ is given in Table 1 to specify the classification of the plant dynamics. At first step, an LQG controller is designed for the nominal plant. Find a controller to minimize

$$
\mathrm{J}=\int_{0}^{\infty}\left(10 \mathrm{y}^{2}+10 \dot{\mathrm{y}}^{2}+\mathrm{u}^{2}\right) \mathrm{dt}
$$

where $y$ is output and $u$ is input of the plant. The optimal control has the from

$$
\mathbf{u}=\mathbf{F x}=\left[\mathbf{f}_{1}, \mathbf{f}_{2}\right] \mathbf{x}
$$

where $\mathrm{x}$ is state vector of the plant. An Kalman filter is constructed to estimate the state vector as follows :

$$
\dot{\hat{\mathbf{x}}}=\mathbf{A} \hat{\mathbf{x}}+\mathbf{B u}+\mathbf{H}(\mathbf{y}-\mathbf{C} \hat{\mathbf{x}})
$$

Filter gain $\mathrm{H}$ should be determined for loop transfer recovery ${ }^{12)}$. From designed $\mathrm{F}$ and $\mathrm{H}, \mathrm{J}(\mathrm{s})$ is obtained according to (5) as follows :

$$
\mathbf{J}(\mathbf{s}) \longleftrightarrow\left[\begin{array}{cc|cc}
-7.321 & 1 & 7.321 & 0 \\
-58.42 & -12.78 & 26.79 & 10 \\
\hline-3.162 & -0.278 & 0 & 1 \\
-1 & 0 & 0 & 0
\end{array}\right]
$$

\begin{tabular}{|c|c|c|c|c|}
\hline Plant & $\mathrm{P}_{\infty}$ & $\mathrm{P}_{\mathrm{ol}}$ & $\mathrm{P}_{\mathrm{lo}}$ & $\mathrm{P}_{11}$ \\
\hline Plant & 1 & 0.5 & 1 & 0.5 \\
\hline dynamics & $\mathrm{s}(1+0.1 \mathrm{~s})$ & $\mathrm{s}(1+0.1 \mathrm{~s})$ & $\mathrm{s}(1+0.4 \mathrm{~s})$ & $\mathrm{s}(1+0.4 \mathrm{~s})$ \\
\hline $\begin{array}{l}\text { Assigned } \\
\text { symbols }\end{array}$ & $(0.0)$ & $(0.1)$ & $(1.0)$ & (1.1) \\
\hline
\end{tabular}

Tuning method for $\mathrm{Q}$ (s) is not objective of this paper. For simplicity, we assume $\mathrm{Q}$ ( $\mathbf{s}$ ) as constant gain $K_{q}$. This tuning part $K_{q}$ of the controller is changed according to symbols from the

Table 1 Plant dynamics and symbolic specification 
network. The value of $K_{q}$ is selected for keeping the response of the nominal control system. Appropriate values of $K_{q}$ to plants : $P_{o o}, P_{o l}, P_{10}, P_{11}$ are $0,-8,-3$, and -9 , respectively. Define $\mathrm{K}_{\mathrm{qoo}}=0, \mathrm{~K}_{\mathrm{qol}}=-8, \mathrm{~K}_{\mathrm{qlo}}=-3, \mathrm{~K}_{\mathrm{ql}}=-9$. Let control systems composed by $\mathrm{J}(\mathrm{s})$ and $\mathrm{K}_{\mathrm{q} i \mathrm{j}}$ be defined by $\mathrm{C}_{\mathrm{ij}}$. In the cases when plant changes from $\mathrm{P}_{\text {oo }}$ to $\mathrm{P}_{\mathrm{ol}}$, unit step responses of control systems are shown in Fig. 6. The damping is not enough in the case of $\left(\mathrm{P}_{\mathrm{ol}}, \mathrm{C}_{\mathrm{oo}}\right)$ and is too fast in the case of $\left(P_{o o}, C_{o}\right)$. Fig. 7 shows the similar results for the change from $P_{o o}$ to $P_{11}$. For the classification of the plant dynamics, we set inputs (data sets S1 and S2 in Section 3) and desired output of the neural network; the outputs of the neural net are the binary valued symbols corresponding to the plant dynamics (See Table 1). After several trials of classifying with neural network, we understood that 30 nodes at hidden level are suitable for both data sets S 1 and S 2 (See Section 3). The learning curve of neural network for classifying is shown in Fig. 8 and Fig. 9. Data set $\mathrm{S} 2$ gives higher recognition rate after 2000 iterations. In most actual cases, state variables are not measurable ; therefore, data set S 2 is preferable. The task of the classification for the neural network is not so
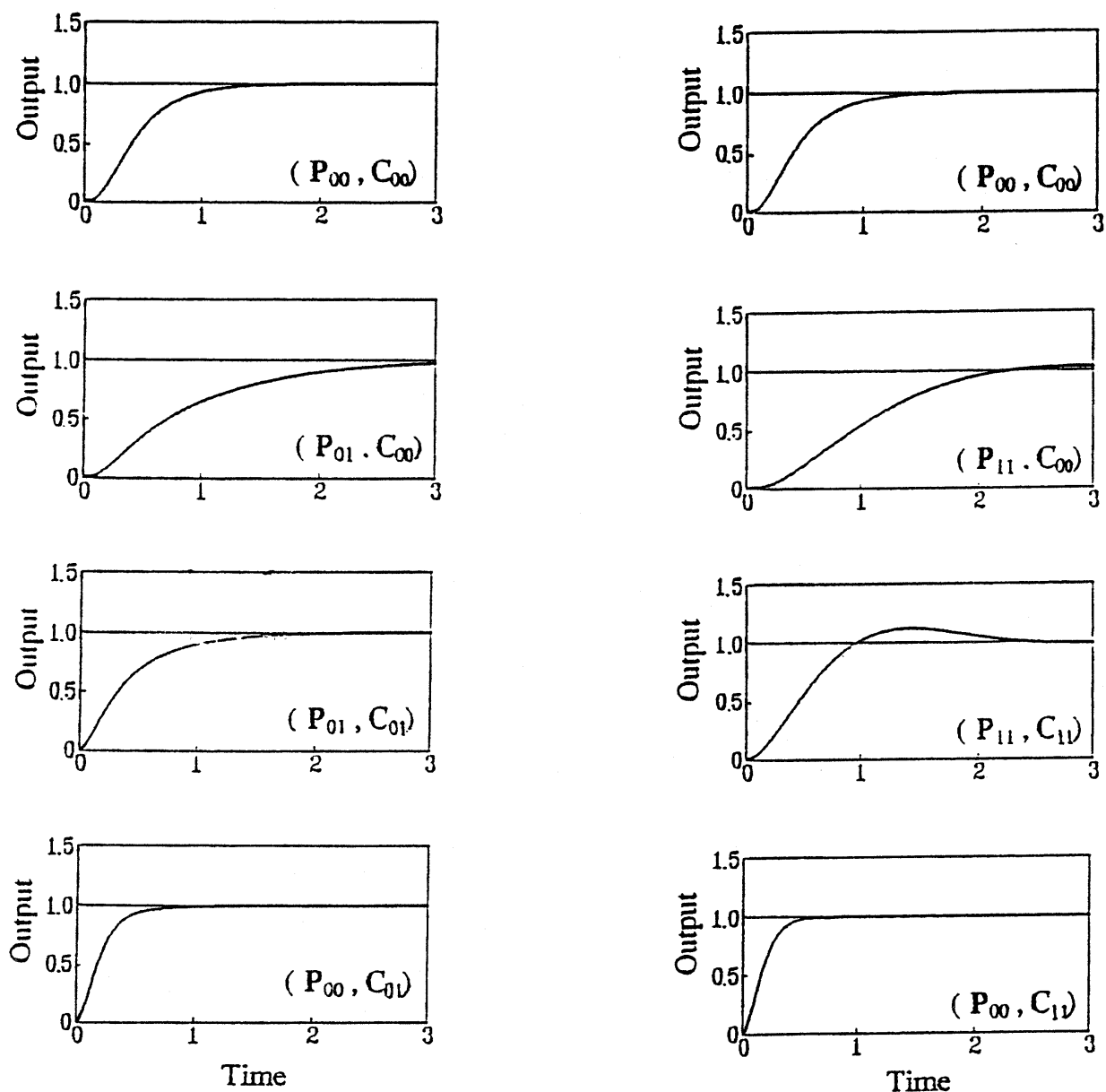

Fig. 6 Unit step responses of each control system (from $\mathrm{P}_{\infty}$ to $\mathrm{P}_{11}$ )

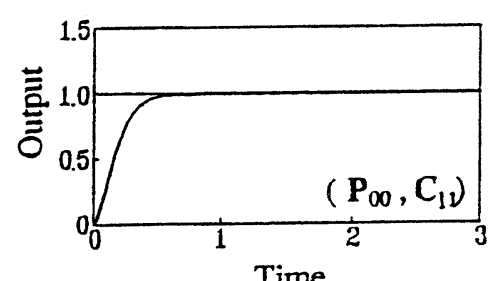

Fig. 7 Unit step responses of each control system (from $\mathrm{P}_{\infty}$ to $\mathrm{P}_{11}$ ) 


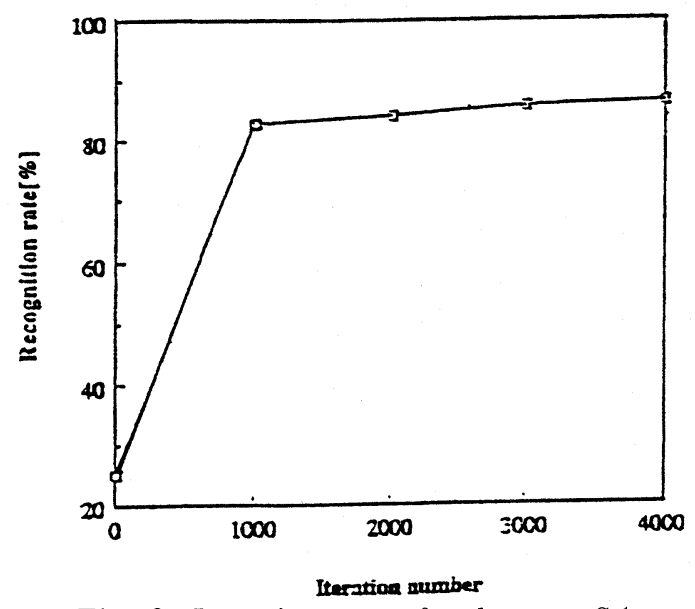

Fig. 8 Learning curve for data set S 1

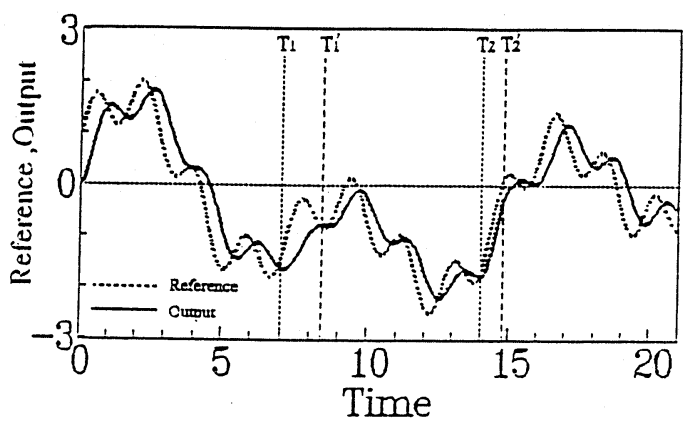

Fig. 10 (a) Reference and plant output with adaptive scheme $\left(\mathrm{P}_{\infty}(\mathrm{s}) \rightarrow \mathrm{P}_{\mathrm{ol}(\mathrm{s})} \rightarrow \mathrm{P}_{\infty}(\mathrm{s})\right.$, $\mathrm{T}_{1}$ : sudden change of plant dynamics, $\mathrm{T}_{2}$ : recovery of plant dynamics, $\mathrm{T}_{1}{ }^{\prime}: \mathrm{de}$ tection of the change by neural network, $\mathrm{Y}_{2}^{\prime}$ : detection of the recovery by neural network)

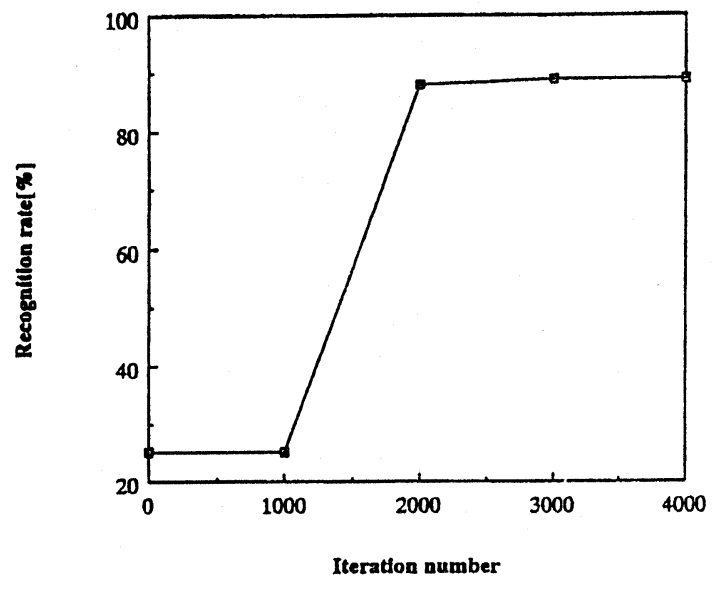

Fig. 9 Learning curve for data set S 2

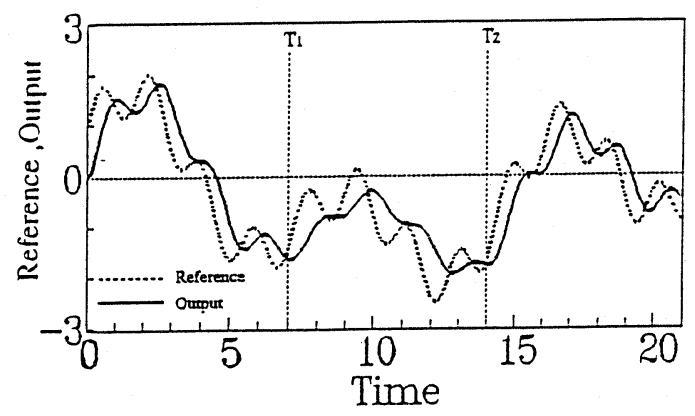

Fig. 10 (b) Reference and plant outputwithout adaptive scheme $\left(\left(\mathrm{P}_{\infty \mathrm{s}(\mathrm{s})} \rightarrow \mathrm{P}_{\mathrm{ol}(\mathrm{s})} \rightarrow \mathrm{P}_{\mathrm{o}(\mathrm{s})}\right.\right.$, $\mathrm{T}_{1}$ : sudden change of plant dynamics, $\mathrm{T}_{2}$ : recovery of plant dynamics)

easy because there are a few parameters in the training stage whose meanings are not clear to the learning with back-propagation. We tried several parameters, but we could not realized the perfect classification. When we apply the trained neural network to adaptive control system, we can modify the decision process for classifying to obtain higher recognition rate as follows: The time series for the inputs of the neural network are checked during a short time interval, then the numeric-tosymbolic conversion is done by the neural network if the checked time are classified as one plant dynamics with the majority. A typical example with this modification is shown in Fig. 10 in the case of change from $P_{o o}$ to $P_{o l}$ and vice versa. Fig. 10 also shows the result in the same condition without the adaptive scheme. Note that the tuning of controller do good on recovering the gain in the response. We obtained the similar results in another changes of plant dynamics. 


\section{Conclusions}

A new control sheme has been proposed for adapting to the varying environment of systems in this paper. The control scheme is based on the parametrization of all stabilizing controllers. The parametrization has been extended to the case of systems which contain numeric-to-symbolic converters. The numeric-to-symbolic converter detects the change of the plant dynamics and selects an appropriate tuning part of the controller. It is shown that the control scheme of this paper is a relatively easy task in comparison with traditional adaptive control scheme.

\section{References}

1) Anderson, B. D. O. : Automatica, 21, 3, 247 (1985).

2) Wittenmark, B. and Astrom, K. : Automatica, 20, 5, 595 (1984).

3) Mareels, I. M. Y. and Bitmead, R. R. : Automatica, 22, 6, 641 (1986).

4) Cassandras, C. G. and Ramadge, P. J. : IEEE Control Systems Magazine, 10, 4, 66 (1990).

5) Li, Y. F. and Lau, C. C. : IEEE Control Systems Magazine, 9, 3, 65 (1989).

6) Kraft, L. G. and Gampagna, D. P. : IEEE Control Systems Magazine, 10, 3, 36 (1990).

7) Vidyasager, M. : Control Systems Synthesis, MIT Press, Cambridge, Massachusetts, (1985).

8) Tay, T. T. and Moore, J. B. : Prep. 8th IFAC/IFROS Symposium on Identification and System Parameter Estimation, 1, 149, (1988).

9) Obinata, G. and Moore, J. B. : Systems and Control Letters, 10, 5, 333 (1988).

10) Obinata, G. : J. Socie. Inst. Contr. Engr., Japan, 28, 11, 988 (1989).

11) Passino, K. M., Sartori, M. A. and Antsakils, P. J. : IEEE Control Systems Magazine, 9, 3, 44 (1989).

12) Stein, G. and Athans, M. : IEEE Trans. Automat. Contr., 32, 2, 105 (1987). 